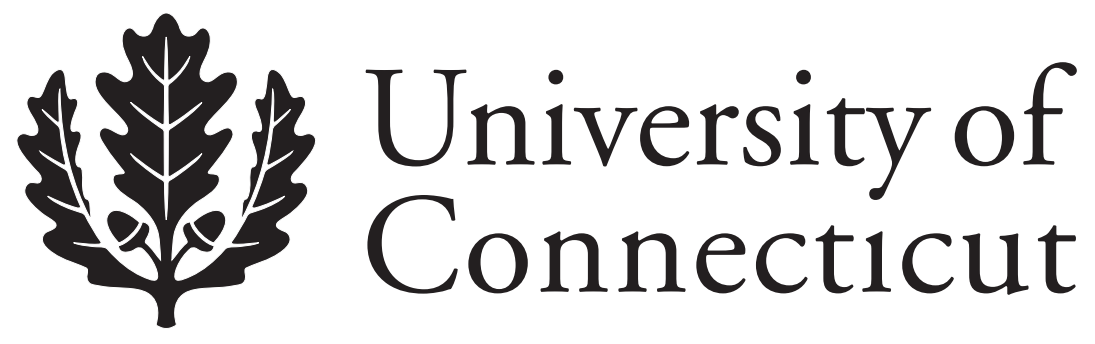

Department of Economics Working Paper Series

\title{
Recognizing One-Dimensional Euclidean Preference Profiles
}

Vicki Knoblauch

University of Connecticut

Working Paper 2008-52R

August 2008

341 Mansfield Road, Unit 1063

Storrs, CT 06269-1063

Phone: (860) 486-3022

Fax: (860) 486-4463

http://www.econ.uconn.edu/

This working paper is indexed on RePEc, http://repec.org/ 


\begin{abstract}
A preference profile has a one-dimensional Euclidean representation if it can be derived from an arrangement of individuals and alternatives on a line, with each individual preferring the nearer of each pair of alternatives. We provide a polynomial-time algorithm that determines whether a given preference profile has a one-dimensional Euclidean representation and, if so, constructs one. This result has electoral and mechanism design applications.
\end{abstract}

Journal of Economic Literature Classification: D11, D72

Keywords: spatial elections, preference representation, mechanism design 


\section{Introduction.}

We address the problem of determining whether a given preference profile has a onedimensional Euclidean representation. We will adopt the language of elections to introduce the problem. In that setting the problem is to determine whether voter preferences over candidates could have been formed solely on the basis of the candidates' and voters' positions on a single issue. More exactly, given a finite set of candidates, a finite electorate and a voter preference profile in the form of a linear ordering of candidates for each voter, is there an arrangement of the candidates and the voters on a line such that for any voter $v$ and candidates $c$ and $d, v$ prefers $c$ to $d$ if and only if the distance from $v$ to $c$ is less than the distance from $v$ to $d$ ?

Here the problem splits in two. In the first version of the problem, voters are allowed to have different perceptions of distance. In particular, for points $c, d$ and $e$ in $\mathbf{R}$ with $d$ between $c$ and $e$, two voters may disagree on whether $d$ is nearer $c$ or $e$, but must agree that $c$ is nearer $d$ than $e$. A spatial representation as described above, in which voters are allowed to have different perceptions of distance, is called a convex representation in $\mathbf{R}$. Equivalently, a convex representation in $\mathbf{R}$ is an arrangement of the candidates alone on a line in such a way that for every voter $v$ and candidate $c$, there is a convex set in $\mathbf{R}$, that is, an interval, that contains those candidates and only those candidates who are weakly preferred to $c$ by $v$.

Convex representations in $\mathbf{R}$ have also been called qualitative scales (Coombs, 1964), and preferences convexly representable in $\mathbf{R}$ have been called ordinally single-peaked preferences (Brams et al, 2002).

Bogomolnaia and Laslier (2007) provide a surprisingly simple answer for the 2-dimensional version of the above question: every preference profile is consistent with voter preferences being formed on the basis of candidates' positions on two issues. In other words, every preference profile has a convex representation in $\mathbf{R}^{\mathbf{2}}$.

Bartholdi and Trick (1986) produced a polynomial time algorithm to determine whether a given voter preference profile has a convex representation in $\mathbf{R}$. Ballester and Haeringer (2007) then presented a simple characterization of convex representability in 
$\mathbf{R}$; they showed that a convex representation in $\mathbf{R}$ exists if and only if the given voter preference profile does not contain as a subprofile either of two examples, one involving three voters and three candidates, and one involving two voters and four candidates.

The second version of the problem differs from the first version only in that voters are required to share a common perception of distance. A spatial representation in $\mathbf{R}$ in which voters are required to share a common perception of distance is called a Euclidean representation in $\mathbf{R}$. Euclidean representations in $\mathbf{R}$ have also been called quantitative scales (Coombs, 1964); and preferences that have Euclidean representations in $\mathbf{R}$ have been called cardinally single-peaked preferences (Brams et al, 2002). Bogomolnaia and Laslier (2007) obtain some interesting results concerning Euclidean representations in higher dimensions. Their results relevant to our problem appear in their Proposition 15, which in dimension 1 asserts the existence of a Euclidean representation in $\mathbf{R}$ if the number of voters is at most two and the number of candidates is at most three, and also provides a two voter, four candidate example with no Euclidean representation in $\mathbf{R}$.

We will construct an algorithm that in polynomial time determines whether a given voter preference profile possesses a Euclidean representation in $\mathbf{R}$ and, if so, constructs such a representation. Here, "in polynomial time" means in a number of steps that is polynomial in the number of candidates.

The algorithm proceeds in five steps. Step 1 checks to see that there aren't too many distinct voters for the given number of candidates. Step 2 constructs a convex representation in $\mathbf{R}$ for the given preference profile, if one exists. Starting with this convex representation, Step 3 reorders a certain subset $B$ of candidates in preparation for Step 4, which solves a linear programming problem to construct a Euclidean representation in $\mathbf{R}$ for the given preferences restricted to $B$, if one exists. Step 5 extends the Euclidean representation to the full preference profile.

Laslier (2003) uses multivariate statistical analysis methods, in particular, Principal Component Analysis (PCA), to study the structure of preference profiles. Since PCA is used to represent preference profiles in low dimensional Euclidean space, it is natural to conjecture that the 1-dimensional PCA representation may in fact provide a 1-dimensional Euclidean representation, whenever one exists. Unfortunately, this is not in fact the case. 
For example, suppose $a, b$ and $c$ are alternatives and 1 and 2 are individuals with preferences $a b c$ and $b c$ a respectively. This preference profile has a 1-dimensional Euclidean representation with $a<b<c$, the order unique up to mirror image. However, the 1dimensional PCA representation, which can be found by following the directions of Laslier (1996, pp 115-116), identifies $b$ and $c$. Of course the general question of whether some other technique of multivariate analysis always provides 1-dimensional Euclidean representations remains open.

Eguia (2008) has actually characterized preference profiles that possess $k$-dimensional Euclidean representations. Unfortunately, the 1-dimensional version of his results does not provide the polynomial time check desired, or even a check in finite time, since his characterizations include a continuum of conditions that must be satisfied by a given preference profile.

Besides the previously discussed application to elections, our results have a mechanism design application. Suppose you are planning a community along a stretch of road. You plan to build homes and several amenities, such as a gym, a grocery store, a bowling alley, etc. Each prospective home-buyer has ranked the amenities from likely-to-be-used-mostoften to likely-to-be-used-least-often. You would like to know if it is possible to place the homes and amenities on the highway so that each homeowner is nearest his top-ranked amenity, second nearest his second-ranked amenity, etc. Since in this context homeowners will have the same perception of distance, we are looking for a Euclidean representation in $\mathbf{R}{ }^{*}$

The rest of the paper is organized as follows. Section 2 consists of preliminaries. Section 3 contains our result on Euclidean representations in $\mathbf{R}$. Section 4 contains some concluding remarks.

\section{Preliminaries.}

* Is there any meaningful interpretation for a convex representation in $\mathbf{R}$ even though homeowners have a common perception of distance? Yes, there is. In a convex representation, no homeowner ever has to drive past a less-often used amenity to get to a more-often used amenity. 
Let $I$ be a finite set of voters or, more generally, individuals. Let $A=\left\{a^{j}\right\}_{j \in J}$ be a finite set of candidates or, more generally, a finite set of distinct alternatives, indexed by $J$, a finite set of positive integers. We assume for convenience that no two voters have identical preferences. Let $R=\left(R_{i}\right)_{i \in I}$ be an ordered $|I|$-tuple of distinct linear orders on A. A linear order $R_{i}$ on $A$ is a complete, transitive, antisymmetric binary relation on $A$. The expression $a^{j} R_{i} a^{k}$ can be read " $i$ weakly prefers $a^{j}$ to $a^{k}$." Alternative $a^{j} \in A$ is $R_{i}$-minimal $\left(R_{i}\right.$-maximal) if $a^{k} R_{i} a^{j}\left(a^{j} R_{i} a^{k}\right)$ for all $a^{k} \in A$. A linear order is essentially a ranking of alternatives from the most preferred $\left(R_{i}\right.$-maximal $)$ to the least preferred $\left(R_{i^{-}}\right.$ minimal). Then $(I, A, R)$ is a profile of linear orders.

For $X \subseteq \mathbf{R}^{\mathbf{d}}, \operatorname{co}(X)$ is the convex hull of $X$ in $\mathbf{R}^{\mathbf{d}}$.

Definition 1. A convex representation in $\mathbf{R}^{\mathbf{d}}$ for profile $(I, A, R)$ is a set $X=\left\{x^{j}\right\}_{j \in J} \subseteq$ $\mathbf{R}^{\mathbf{d}}$ such that for all $i \in I$ and $x^{k} \in X$, the upper contour set $U_{i}\left(x^{k}\right):=\left\{x^{j}: a^{j} R_{i} a^{k}\right\}$ satisfies $U_{i}\left(x^{k}\right)=\operatorname{co}\left(U_{i}\left(x^{k}\right)\right) \cap X$.

Definition 2. A Euclidean representation in $\mathbf{R}^{\mathbf{d}}$ for profile $(I, A, R)$ is an ordered pair $(X, W)$ with $X \cup W=\left\{x^{j}\right\}_{j \in J} \cup\left\{w^{i}\right\}_{i \in I} \subseteq \mathbf{R}^{\mathbf{d}}$ such that for $i \in I$ and distinct $a^{j}, a^{k} \in A$, $a^{j} R_{i} a^{k}$ if and only of $\rho\left(x^{j}, w^{i}\right)<\rho\left(x^{k}, w^{i}\right)$, where $\rho$ is Euclidean distance.

Definition 3. A profile of linear orders $(I, A, R)$ is 3,3-twisted if there exist $b, c, d \in I$ and distinct $a^{p}, a^{q}, a^{r} \in A$ such that, among $a^{p}, a^{q}$ and $a^{r}, a^{p}$ is $R_{b^{-m i n i m a l}} a^{q}$ is $R_{c^{-m i n i m a l}}$ and $a^{r}$ is $R_{d}$-minimal.

Definition 4. A profile of linear orders $(I, A, R)$ is 2,4-twisted if there exist $b, c \in I$ and distinct $a^{p}, a^{q}, a^{r}, a^{s} \in A$ such that $a^{r} R_{b} a^{q} R_{b} a^{p}, a^{p} R_{c} a^{q} R_{c} a^{r}, a^{s} R_{b} a^{q}$ and $a^{s} R_{c} a^{q}$.

Proposition 1. (Ballester and Haeringer, 2007) A profile of linear orders $(I, A, R)$ has a convex representation in $\mathbf{R}$ if and only if it is neither 3,3-twisted nor 2,4-twisted.

A family of problems $(Q(n))$ is an infinite sequence of collections of problems. Consider an algorithm that solves $(Q(n))$, that is, an algorithm that solves every $Q \in \cup_{n=1}^{+\infty} Q(n)$. Such an algorithm is a polynomial-time algorithm if there is a polynomial $P$ such that for every positive integer $n$, the algorithm solves each $Q \in Q(n)$ in at most 
$P(n)$ steps. In general, a polynomial-time algorithm can be usefully implemented on a computer, an algorithm that takes an exponential number of steps cannot.

\section{Euclidean Representations in R.}

We now construct an algorithm that inputs a voter preference profile $(I, A, R)$ and outputs a Euclidean representation $(X, W)$ with $X \cup W=\left\{x^{j}\right\}_{j \in J} \cup\left\{w^{i}\right\}_{i \in I} \subseteq \mathbf{R}$ or announces that no Euclidean representation in $\mathbf{R}$ exists.

Step 1. First check to see that $|I| \leq\left(\begin{array}{c}|A| \\ 2\end{array}\right)+1$. This is a necessary condition, since, if $(X, W)$ is a Euclidean representation in $\mathbf{R}$ for $(I, A, R)$ and $w^{b}<w^{c}$ it follows from the fact that voters have distinct preferences that there must exist $x^{p}$ and $x^{q}$ such that $w^{b}<\left(x_{p}+x_{q}\right) / 2<w^{c}$. Since there are $\left(\begin{array}{c}|A| \\ 2\end{array}\right)$ candidate-pair midpoints, there can be at $\operatorname{most}\left(\begin{array}{c}|A| \\ 2\end{array}\right)+1$ voters.

Step 2. Use Proposition 1 to determine whether $(I, A, R)$ possesses a convex representation in $\mathbf{R}$. If not, $(I, A, R)$ possesses no Euclidean representation in $\mathbf{R}$. If so, construct a convex representation $X$ in $\mathbf{R}$ for $(I, A, R)$ using the method of Ballester and Haeringer (2007) or Bartholdi and Trick (1986).

Next, in order to establish and exploit the (limited) uniqueness of Euclidean representations in $\mathbf{R}$, we need to reindex the candidates. Suppose $a^{|A|}, a^{|A|-1}, \ldots, a^{r+1}$ have been chosen for $1 \leq r \leq|A|$. (If $r=|A|$, this means the reindexing has not yet begun.) Choose $a^{r}$ to satisfy

$a^{r}$ is $R_{i}$-minimal in $A-\left\{a^{|A|}, a^{|A-1|}, \ldots, a^{r+1}\right\}$ for some $i$ and, if possible, such that there exists $a^{k}$ with $a^{r} R_{i}$-minimal in $A-\left\{a^{|A|}, a^{|A-1|}, \ldots, a^{r+1}, a^{k}\right\}$ for all $i$

Notice that the construction is not in general unique. By our use of (1) in the construction,

$$
\text { for all } j, a^{j} \text { is } R_{i} \text {-minimal in }\left\{a^{1}, a^{2}, \ldots, a^{j}\right\} \text { for some } i
$$

Now partition $A$ into $t$ sets

$$
\begin{aligned}
& \left\{A_{1}=\left\{a^{k_{1}}=a^{1}, a^{2}, \ldots, a^{k_{2}-1}\right\}, A_{2}=\left\{a^{k_{2}}, a^{k_{2}+1}, \ldots, a^{k_{3}-1}\right\},\right. \\
& \left.\ldots, A_{t}=\left\{a^{k_{t}}, a^{k_{t}+1}, \ldots, a^{|A|}\right\}\right\}
\end{aligned}
$$


defined inductively by

$k_{1}=1, k_{l+1}$ is the smallest $j>k_{l}$, such that $a^{j}$ is $R_{i}-$ minimal in $\left\{a^{1}, a^{2}, \ldots, a^{j}\right\}$ for all $i$

Lemma 1. If $(I, A, R)$ has a convex representation in $\mathbf{R}$ and $\left|A_{l}\right|>1$, then, up to order in $\mathbf{R}$, there are exactly two convex representations in $\mathbf{R}$ for $\left(I, A_{l},\left.R\right|_{A_{l}}\right)$, and these two convex representations are oppositely ordered.

Proof. Suppose $\left|A_{l}\right|>1$ and $Z_{l}=\left\{z^{k_{l}}, z^{k_{l}+1}, \ldots, z^{k_{l+1}-1}\right\}\left(\right.$ or $\left\{z^{k_{l}}, z^{k_{l}+1}, \ldots, z^{|A|}\right\}$ if $l=$ $t)$ is a convex representation in $\mathbf{R}$ for $\left(I, A_{l},\left.R\right|_{A_{l}}\right)$. Then $\left\{z^{k_{l}}, z^{k_{l}+1}\right\}$ and $\left\{-z^{k_{l}},-z^{k_{l}+1}\right\}$ are convex representations in $\mathbf{R}$ for $\left(I,\left\{a^{k_{l}}, a^{k_{l}+1}\right\},\left.R\right|_{\left\{a^{\left.k_{l}, a^{k_{l}+1}\right\}}\right.}\right)$ and they are oppositely ordered in $\mathbf{R}$. If $\left|A_{l}\right|>2$, by (2) and (3) both $a^{k_{l}+2}$ and $a^{q}$ are $R_{i}$-minimal in $\left\{a^{k_{l}}, a^{k_{l}+1}, a^{k_{l}+2}\right\}$ for some $i$, where $a^{q} \neq a^{k_{l}+2}$. Then, both $z^{k_{l}+2}$ and $z^{q}$ are extrema of $\left\{z^{k_{l}}, z^{k_{l}+1}, z^{k_{l}+2}\right\}$. Therefore the order of $\left\{z^{k_{l}}, z^{k_{l}+1}\right\}$ in $\mathbf{R}$ uniquely determines the order of $\left\{z^{k_{l}}, z^{k_{l}+1}, z^{k_{l}+2}\right\}$ in $\mathbf{R}$. In other words, up to order in $\mathbf{R}$ there are exactly two convex representations in $\mathbf{R}$ for $\left.\left(I,\left\{a^{k_{l}}, a^{k_{l}+1}, a^{k_{l}+2}\right\},\left.R\right|_{\left\{a^{k_{l}}\right.}, a^{k_{l}+1}, a^{k_{l}+2}\right\}\right): \quad\left\{z^{k_{l}}, z^{k_{l}+1}, z^{k_{l}+2}\right\}$ and $\left\{-z^{k_{l}},-z^{k_{l}+1},-z^{k_{l}+2}\right\}$. Continue adding on one $a^{j}$ at a time until the conclusion holds for $A_{l}$

Our description of Step 3 requires the following notation. Let $B=\cup_{\left|A_{l}\right|>1} A_{l}$. Let $A_{l 1}, A_{l 2} \ldots, A_{l s}$ be the subsequence of $A_{1}, A_{2}, \ldots, A_{t}$ containing all $A_{l} \subseteq B$. For any set $Z \subseteq \mathbf{R}$, let $-Z=\{-z: z \in Z\}$.

Step 3. From Step 2 we have a convex representation $X \subseteq \mathbf{R}$ for $(I, A, R)$ and therefore a convex representation $Z \subseteq \mathbf{R}$ for $\left(I, B,\left.R\right|_{B}\right)$. We will define a linear order $\leq$ on $Z$ (not in general the order inherited by $Z$ from $\mathbf{R}$ under less-than-or-equal) such that the order of candidates in every Euclidean representation in $\mathbf{R}$ for $\left(I, B,\left.R\right|_{B}\right)$ agrees with $\leq$ or its inverse. Clearly, this agreement condition places no restrictions on the linear order $(Z, \leq)$ if $\left(I, B,\left.R\right|_{B}\right)$ possesses no Euclidean representation in $\mathbf{R}$.

Let $Z_{l 1}=\left\{z^{j}: a^{j} \in A_{l 1}\right\}$. Using Lemma 1, without loss of generality we can let $\leq$ on $Z_{l 1}$ be the order on $Z_{l 1}$ inherited from $\mathbf{R}$ ordered by less than or equal (in short, let $\leq$ on 
$Z_{l 1}$ agree with $\left.Z_{l 1} \subseteq \mathbf{R}\right)$. By Lemma 1 we must define $\leq$ on $Z_{l 2}$ to agree with $Z_{l 2} \subseteq \mathbf{R}$ or to be ordered oppositely to $Z_{l 2} \subseteq \mathbf{R}$.

Question 1. Is there a Euclidean representation in $\mathbf{R}$ for $\left(I, B,\left.R\right|_{B}\right)$ with $Z_{l 1}$ ordered by $\leq$, with $Z_{l 2}$ ordered like $Z_{l 2} \subseteq \mathbf{R}$, and such that $\frac{z^{k_{l 1}}+z^{k_{l 1}+1}}{2} \leq \frac{z^{k_{l 2}}+z^{k_{l 2}+1}}{2}$ ? Without loss of generality, assume $z^{k_{l 1}}<z^{k_{l 1}+1}$ and $z^{k_{l 2}}<z^{k_{l 2}+1}$. By (2) and (3), choose $b, c \in I$ such that $a^{k_{l 1}} R_{b} a^{k_{l 1}+1}$ and $a^{k_{l 2}+1} R_{c} a^{k_{l 2}}$. A "yes" answer to Question 1 would imply $a^{k_{l 2}} R_{b} a^{k_{l 2}+1}$ and $a^{k_{l 1}+1} R_{c} a^{k_{l 1}}$.

Question 2. Same question, but with $\frac{z^{k_{l 2}+z^{k}{ }^{2}+1}}{2}<\frac{z^{k_{l 1}}+z^{k_{l 1}+1}}{2}$. By (2) and (3), choose $d, e \in I$ such that $a^{k_{l 1}+1} R_{d} a^{k_{l 1}}$ and $a^{k_{l 2}} R_{e} a^{k_{l 2}+1}$. A "yes" answer to Question 2 would imply $a^{k_{l 2}+1} R_{d} a^{k_{l 2}}$ and $a^{k_{l 1}} R_{e} a^{k_{l 1}+1}$

If $\operatorname{not}\left(a^{k_{l 2}} R_{b} a^{k_{l 2}+1}\right.$ and $\left.a^{k_{l 1}+1} R_{c} a^{k_{l 1}}\right)$ and $\operatorname{not}\left(a^{k_{l 2}+1} R_{d} a^{k_{l 2}}\right.$ and $\left.a^{k_{l 1}} R_{e} a^{k_{l 1}+1}\right)$, then the answers to Questions 1 and 2 are "no" and "no." There is no Euclidean representation in $\mathbf{R}$ for $\left(I, B,\left.R\right|_{B}\right)$ with $Z_{l 1}$ ordered by $\leq$ and $Z_{l 2}$ ordered like $Z_{l 2} \subseteq \mathbf{R}$. Therefore we adopt the only remaining alternative and define $\leq$ on $Z_{l 2}$ to be ordered oppositely to $Z_{l 2} \subseteq \mathbf{R}$. This guarantees that in every Euclidean representation in $\mathbf{R}$ for $\left(I, B,\left.R\right|_{B}\right)$, the order of $Z_{l 1}$ and the order of $Z_{l 2}$ both agree with $\leq$ or both agree with its inverse.

On the other hand, if $a^{k_{l 2}} R_{b} a^{k_{l 2}+1}$ and $a^{k_{l 1}+1} R_{c} a^{k_{l 1}}$ we ask a third question.

Question 3. Is there a Euclidean representation in $\mathbf{R}$ for $\left(I, B,\left.R\right|_{B}\right)$ with $Z_{l 1}$ ordered by $\leq$ and $Z_{l 2}$ ordered oppositely to $Z_{l 2} \subseteq \mathbf{R}$ ? If so we have $z^{k_{l 1}}<z^{k_{l 1}+1}$ and $z^{k_{l 2}+1}<z^{k_{l 2}}$. If $\frac{z^{k_{l 1}}+z^{k_{l 1}+1}}{2} \leq \frac{z^{k_{l 2}}+z^{k_{l 2}+1}}{2}$, then by $a^{k_{l 1}} R_{b} a^{k_{l 1}+1}, a^{k_{l 2}+1} R_{b} a^{k_{l 2}}$, a contradiction. If $\frac{z^{k_{l 2}}+z^{k_{l 2}+1}}{2}<\frac{z^{k_{l 1}}+z^{k_{l 1}+1}}{2}$, then by $a^{k_{l 2}+1} R_{c} a^{k_{l 2}}, a^{k_{l 1}} R_{c} a^{k_{l 1}+1}$, a contradiction.

Therefore the answer to Question 3 is "no." We adopt the only remaining alternative and define $\leq$ on $Z_{l 2}$ to agree with $Z_{l 2} \subseteq \mathbf{R}$. This guarantees that in every Euclidean representation in $\mathbf{R}$ for $\left(I, B,\left.R\right|_{B}\right)$, the order of $Z_{l 1}$ and the order of $Z_{l 2}$ both agree with $\leq$ or both agree with its inverse.

The final case, $a^{k_{l 2}+1} R_{d} a^{k_{l 2}}$ and $a^{k_{l 1}} R_{e} a^{k_{l 1}+1}$, similarly leads us to define $\leq$ on $Z_{l 2}$ to agree with $Z_{l 2} \subseteq \mathbf{R}$.

Next use $\leq$ on $Z_{l 2}$ to define $\leq$ on $Z_{l 3}$, use $\leq$ on $Z_{l 3}$ to define $\leq$ on $Z_{l 4}$, etc.

Now extend $\leq$ on $Z_{l 1}$ and $\leq$ on $Z_{l 2}$ to $\leq$ on $Z_{l 1} \cup Z_{l 2}$ so that the order of $Z_{l 1} \cup Z_{l 2}$ 
in every Euclidean representation in $\mathbf{R}$ for $\left(I, B,\left.R\right|_{B}\right)$ agrees with $\leq$ on $Z_{l 1} \cup Z_{l 2}$ or its inverse as follows. By (2), (3) and the fact that a candidate least preferred by any voter must give rise to an extremum of a convex representation, $z^{k_{l 2}}$ and $z^{k_{l 2}+1}$ must be extrema of $\leq$ on $Z_{l 1} \cup\left\{z^{k_{l 2}}, z^{k_{l 2}+1}\right\}$; otherwise $\leq$ on $Z_{l 1} \cup\left\{z^{k_{l 2}}, z^{k_{l 2}+1}\right\}$ cannot agree with the order of $Z_{l 1} \cup\left\{z^{k_{l 2}}, z^{k_{l 2}+1}\right\}$ in a convex representation in $\mathbf{R}$ for $\left(I, B,\left.R\right|_{B}\right)$, and therefore cannot agree with the order in a Euclidean representation in $\mathbf{R}$ for $\left(I, B,\left.R\right|_{B}\right)$. Then $\leq$ on $Z_{l 1}$ and on $Z_{l 2}$ determines $\leq$ on $Z_{l 1} \cup\left\{z^{k_{l 2}}, z^{k_{l 2}+1}\right\}$. If $\left|A_{l 2}\right| \geq 3, z^{k_{l 2}+2}$ and either $z^{k_{l 2}}$ or $z^{k_{l 2}+1}$ must be extrema of $\leq$ on $Z_{l 1} \cup\left\{z^{k_{l 2}}, z^{k_{l 2}+1}, z^{k_{l 3}+1}\right\}$ so that $\leq$ on $Z_{l 1}$ and on $Z_{l 2}$ determines $\leq$ on $Z_{l 1} \cup\left\{z^{k_{l 2}}, z^{k_{l 2}+1}, z^{k_{l 2}+2}\right\}$. Continuing in this way, we define $\leq$ on $Z_{l 1} \cup Z_{l 2}$, then use $\leq$ on $Z_{l 2}$ and $Z_{l 3}$ to define $\leq$ on $Z_{l 1} \cup Z_{l 2} \cup Z_{l 3}$, etc. We pass the linear order $(Z, \leq)$ to Step 4 , considering $Z$ as simply an abstract set on which a linear order has been defined.

Step 4. From Step 3 we have $Z=\left\{z^{j}: a^{j} \in B\right\}$ and a linear order $\leq$ on $Z$ such that the order of candidates in every Euclidean representation in $\mathbf{R}$ for $\left(I, B,\left.R\right|_{B}\right)$ agrees with $\leq$ or its inverse. We now want to think of $Z$ as a subset of $\mathbf{R}$ that is not completely specified, but such that the order of $Z$ in $\mathbf{R}$ is in agreement with the linear order $\leq$ defined in Step 3. We can completely specify $Z$ and also define $W=\left\{w^{1}, w^{2}, \ldots, w^{|I|}\right\} \subseteq \mathbf{R}$ so that $(Z, W)$ is a Euclidean representation in $\mathbf{R}$ for $\left(I, B,\left.R\right|_{B}\right)$ if and only if there is a solution in real values to the following system of linear inequalities:

(1) all inequalities $z^{p}<z^{q}$ from the given linear order $(Z, \leq)$, passed from Step 3 and

(2) $\frac{\left(z^{r}+z^{s}\right)}{2}<\frac{\left(z^{p}+z^{q}\right)}{2}$ if $z^{p}, z^{q}, z^{r}, z^{s} \in Z, z^{p}<z^{q}, z^{r}<z^{s}$ and there exists $b \in I$ such that $z^{p} R_{b} z^{q}$ and $z^{s} R_{b} z^{r}$.

If a solution $Z$ exists, $W$ is defined from $Z$ as follows, If $b \in I, z^{p}, z^{q} \in Z$ and $z^{p}<z^{q}$, then $w^{b}<\frac{z^{p}+z^{q}}{2}$ if $z^{p} R_{b} z^{q}$ and $w^{b}>\frac{z^{p}+z^{q}}{2}$ if $z^{q} R_{b} z^{p}$.

Notice that the number of unknowns in the system of inequalities defining $Z$ is $|B| \leq|A|$ and the number of inequalities is less than $|B|^{2}+|I||B|^{4} \leq|B|^{2}+\left(\left(\begin{array}{c}|B| \\ 2\end{array}\right)+1\right)|B|^{4} \leq$ $|B|^{6} \leq|A|^{6}$

At the end of Step 4, we have arrived at one of two possible outcomes.

The first possibility is that we have concluded that there is no Euclidean representation in $\mathbf{R}$ for $\left(I, B,\left.R\right|_{B}\right)$ in which the order of candidates agrees with the linear order on $Z$ 
defined in Step 3. Then by Step 3, there is no Euclidean representation in $\mathbf{R}$ for $\left(I, B,\left.R\right|_{B}\right)$, which implies there is no Euclidean representation in $\mathbf{R}$ for $(I, A, R)$.

The second possibility is that we have constructed a Euclidean representation $(Z, W)$ in $\mathbf{R}$ for $\left(I, B,\left.R\right|_{B}\right)$.

We will use the following fact to extend $(Z, W)$ to a Euclidean representation in $\mathbf{R}$ for $(I, A, R)$.

Lemma 2. Suppose $(I, A, R)$ has a convex representation in $\mathbf{R}, i \in I,\left|A_{l}\right|=1, A_{l} \neq A_{m}$ and $a^{r} \in A_{m}$. Then $a^{r} R_{i} a^{k_{l}}$ if and only if $m<l$.

Proof. By (3) $a^{k_{l}}$ is $R_{i}$-minimal in $\left\{a^{1}, a^{2}, \ldots, a^{k_{l}}\right\}$. If $m<l$, then $r<k_{l}$ so that $a^{r} R_{i} a^{k_{l}}$. Now suppose there exists $r, l, m \leq|A|$ and $b \in I$ with $a^{r} \in A_{m}, l<m$ and $a^{r} R_{b} a^{k_{l}}$.

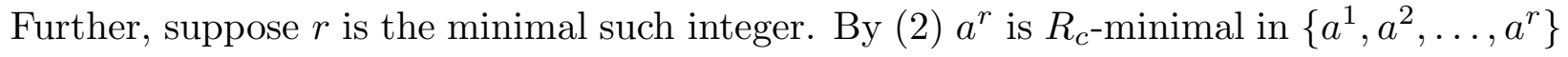
for some $c \in I$. Since $k_{l+1} \leq r$ and since $a^{k_{l}} R_{i} a^{k_{l+1}}$ for all $i$, (which follows from (3)) $a^{k_{l}}$ is not $R_{b}$-minimal in $\left\{a^{1}, a^{2}, \ldots, a^{r}\right\}$. Therefore there exists $p$ with $k_{l}<p<r$ such that $a^{p}$ is $R_{b}$-minimal in $\left\{a^{1}, a^{2}, \ldots, a^{r}\right\}$ and $a^{k_{l}} R_{c} a^{p}$ by the minimality of $r$. Since $a^{k_{l}} R_{c} a^{p} R_{c} a^{r}$, there must be $q$ with $k_{l}<q<r$ and $q \neq p$ such that $a^{k_{l}} R_{c} a^{p} R_{c} a^{q} R_{c} a^{r}$ for some $c$. If there were no such $q$ for all $c$ with $a^{p} R_{c} a^{r}$, we could not have $p<r$ by (1). We also have $a^{k_{l}} R_{b} a^{q}$ by the minimality of $r$.

We now have $a^{k_{l}} R_{c} a^{p} R_{c} a^{q} R_{c} a^{r}$ and $a^{r} R_{b} a^{k_{l}} R_{b} a^{q} R_{b} a^{p}$. Setting $k_{l}=s$, these expressions say $(I, A, R)$ is 2-4 twisted, which together with Proposition 1 contradicts the convex representability in $\mathbf{R}$ of $(I, A, R)$.

Step 5. From Step 4 we have a Euclidean representation $(Z, W)$ in $\mathbf{R}$ for $\left(I, B,\left.R\right|_{B}\right)$, which we now use to construct a Euclidean representation $(X, W)$, in $\mathbf{R}$ for $(I, A, R)$. Suppose $\left|A_{l}\right|=1$. We may have $l<m$ for all $A_{m} \subseteq B, l>m$ for all $A_{m} \subseteq B$, or $m<l<n$ for some $m, n$ with $A_{m}, A_{n} \subseteq B$. We will deal with the third case, which is the most difficult. By Lemma 2, if $a^{p} \in A_{m}, a^{q} \in A_{n}$ and $m<l<n$, then $a^{p} R_{i} a^{k_{l}} R_{i} a^{q}$ for all $i \in I$. We first construct $Y \subseteq \mathbf{R}$ by setting $y^{p}=z^{p}$ for $a^{p} \in B$ such that $p<k_{l}$; by setting $y^{q}>z^{q}$ if $q>k_{l}$ and $z^{q}>z^{p}$ for $a^{p} \in B$ such that $p<k_{l}$; and by setting $y^{q}<z^{q}$ if $q>k_{l}$ and $z^{q}<z^{p}$ for $a^{p} \in B$ such that $p<k_{l}$. 
If in addition we construct $Y$ so that $\left|y^{q}-z^{q}\right|=\left|y^{r}-z^{r}\right|$ whenever $y_{q} \neq z_{q}$ and $y_{r} \neq z_{r}$, then $(Y, W)$ will be a Euclidean representation in $\mathbf{R}$ for $\left(I, B,\left.R\right|_{B}\right)$, since $\frac{\left(y^{q}+y^{r}\right)}{2}=\frac{\left(z^{q}+z^{r}\right)}{2}$ if $y^{q}>z^{q}$ and $y^{r}<z^{r}$ or if $y^{q}=z^{q}$ and $y^{r}=z^{r}$, and since in any other case $y^{q}, y^{r}$ and $\frac{\left(y^{q}+y^{r}\right)}{2}$ will be in the same direction from each voter as were $z^{q}, z^{r}$ and $\frac{\left(z^{q}+z^{r}\right)}{2}$, respectively. Finally, if $\left|y^{q}-z^{q}\right|$ is chosen large enough whenever $y^{q} \neq z^{q}$, then we can define $y^{k_{l}}$ by placing it between $\left\{y^{p}: y^{p}=z^{p}\right\}$ and $\left\{y^{p}: y^{p}>z^{p}\right\}$ (or $\left\{y^{p}: y^{p}<z^{p}\right\}$ if we prefer), in such a way that $\left(Y \cup\left\{y^{k_{l}}\right\}, W\right)$ is a Euclidean representation in $\mathbf{R}$ for $\left(I, B \cup\left\{a^{k_{l}}\right\},\left.R\right|_{B \cup\left\{a^{k_{l}}\right\}}\right)$. Continuing to treat one $a^{k_{l}}$ at a time in this manner, we arrive at $(X, W)=\left(Y \cup\left\{y^{k_{l}}:\left|A_{l}\right|=\right.\right.$ $1\}, W)$, a Euclidean representation in $\mathbf{R}$ for $(I, A, R)$.

It is easy to see that Steps 1, 3 and 5 of our algorithm are accomplished in polynomial time, that is, in a number of steps that is polynomial in $|A|$. Bartholdi and Trick (1986) proved that Step 2 can be acccomplished in polynomial time. We discuss Step 4 in Section 5 .

Finally by our construction, the number of distinct Euclidean representations in $\mathbf{R}$ for a given representable $(I, A, R)$, where two representations are distinct if they order the candidates differently, is $2^{\left|A-B-A_{1}\right|+\delta}$ where $\delta= \begin{cases}1 & \text { if } B \neq \emptyset \\ 0 & \text { if } B=\emptyset\end{cases}$

\section{Concluding Remarks: Linear Programming.}

We note that our application of linear programming is somewhat unusual. Gale (2007) points out that almost all linear programming applications concern consumption or production problems; that is, they involve optimizing over a set of processes that consume or produce a set of goods.

Concerning the complexity of linear programming problems, it is well known that in practice the famous simplex method solves linear programming problems relatively quickly. Borgwardt (1982) and Smale (1983) proved that the average number of steps required by the simplex method is polynomial. However, Klee and Minty (1972) had already demonstrated that for worst-case examples, the simplex method requires an exponential number of steps. Fortunately, Khachiyan (1980) demonstrated that the ellipsoidal method does in fact solve linear programming problems in polynomial time. Since we have a polynomialtime reduction of our problem, determining whether a Euclidean representation in $\mathbf{R}$ exists 
and if so constructing one, to a linear programming problem, and since linear programming problems are solvable in polynomial time, our problem is solvable in polynomial time. 


\section{References}

1. M. Ballester and G. Haeringer, A characterization of single peaked preferences, working paper, 2007.

2. J. Bartholdi III and M. Trick, Stable matching with preferences derived from a psychological model, Operations Research Letters 5 (1986), 165-169.

3. A. Bogomolnaia and J. Laslier, Euclidean preferences, Journal of Mathematical Economics 43 (2007), 87-98.

4. K. H. Borgwardt, The average number of pivot steps required by the simplex method is polynomial, Zeitschrift für Operations Research 7 (1982), 157-177.

5. S. Brams, M. Jones and M. Kilgour, Single-peakedness and disconnected coaltions, Journal of Theoretical Politics 14 (2002), 359-383.

6. C. Coombs, A Theory of Data, New York: John Wiley and Sons, 1964.

7. J. Eguia, Foundations of spatial preferences, working paper, 2008.

8. D. Gale, Linear Programming and the simplex method, Notices of the American Mathematical Society 54 (2007), 364-369.

9. L. G. Khachiyan, Polynomial algorithms in linear programming, USSR Computational Mathematics and Mathematical Physics 20 (1980), 53-72.

10. V. Klee and G. J. Minty, How good is the simplex algorithm? in (O. Shishe, ed), Inequalities III, New York, Academic Press (1972), 159-175.

11. J. F. Laslier, Multivariate description of comparison matrices, Journal of MultiCriteria Decision Analysis 5 (1996), 112-126.

12. J. F. Laslier, Analyzing a preference and approval profile, Social Choice and Welfare 20 (2003), 229-242.

13. S. Smale, On the average number of steps of the simplex method of linear programming, Mathematical Programming 27 (1983), 241-262. 